\title{
Narrow Band Gap Lead Sulfide Hole Transport Layers for Quantum Dot Photovoltaics
}

\author{
Nanlin Zhang, ${ }^{\dagger}$ Darren C. J. Neo, ${ }^{\dagger}$ Yujiro Tazawa, ${ }^{\dagger}$ Xiuting Li,${ }^{\S}$ Hazel E. Assender,$^{\dagger}$ \\ Richard G. Compton, ${ }^{\S} \&$ Andrew A. R. Watt ${ }^{\dagger, *}$ \\ ${ }^{\dagger}$ Department of Materials, University of Oxford, 16 Parks Road, Oxford OX1 3PH, \\ United Kingdom \\ $\S$ Department of Chemistry, Physical \& Theoretical Chemistry Laboratory, University of \\ Oxford, Oxford OX1 3QZ, United Kingdom
}

\begin{abstract}
The band structure of colloidal quantum dot (CQD) bilayer heterojunction solar cells is optimized using a combination of ligand modification and QD band gap control. Solar cells with power conversion efficiencies of up to $9.33 \pm 0.50 \%$ are demonstrated by aligning the absorber and hole transport layers (HTL). Key to achieving high efficiencies is optimizing the relative position of both the valence band and Fermi energy at the CQD bilayer interface. By comparing different band-gap CQDs with different ligands we find that a smaller band gap CQD HTL in combination with a more p-type-inducing CQD ligand is found to enhance hole extraction and hence device performance. We postulate that the efficiency improvements observed are largely due to the synergistic effects of narrower band-gap QDs causing an upshift of valence band
\end{abstract}


position due to 1, 2-ethanedithiol (EDT) ligands and a lowering of the Fermi level due to oxidation.

KEYWORDS quantum dot, solar cell, band alignment optimization, surface treatment, band gap control

\section{INTRODUCTION}

Solar cells based on lead sulfide colloidal quantum dots as the absorber have the potential to provide cheap, flexible, and highly efficient photovoltaic devices. ${ }^{1-2}$ Lead and sulphur are both abundant, low cost materials that can be synthesized into nanocrystal colloids which absorb across the solar spectrum. ${ }^{3-4}$ These colloids can be solutionprocessed into thin films with controlled semiconductor properties suitable for optoelectronic devices. Since the first report of a Schottky junction solar cell in 2005, ${ }^{5}$ power conversion efficiencies (PCE) have increased from less than $1 \%$ to $11.3 \% .^{1,6-7}$

To be a viable solar energy technology improvements in fill factor (FF) and open circuit voltage $\left(\mathrm{V}_{\text {oc }}\right)$ are still required. Recent device improvements have focused on carrier mobility and recombination using a variety of treatments including solid or liquid state ligand exchange, ${ }^{8-11}$ surface passivation, ${ }^{12-14}$ and band alignment engineering ${ }^{2,15-19}$. It is accepted that the chemistry at the CQD surface determines carrier properties as much as the QD's bulk properties. ${ }^{20-21}$ For example Tang et al. ${ }^{12}$ showed that single halide atom passivation reduces the number of trap sites, and lowers the recombination rate of electrons and holes, compared to organic-only counterparts e.g. EDT. Ip et al. ${ }^{14}$ further introduced a hybrid passivation method using atomic and organic ligands together, efficiently reducing the number of trap energy levels in the band gap. 
The alignment of the conduction and valence bands and Fermi level at interfaces is also critical to device performance. Chuang et al. ${ }^{2}$ showed that replacing two layers of tetrabutylammonium iodide (TBAI)-modified PbS with EDT-modified PbS acts to block electrons at the cathode, resulting in a certified efficiency of $8.55 \%$. Brown et al. ${ }^{15}$ tabulated the effects of various ligands on the Fermi levels and valence band positions. Besides different ligands, ${ }^{2,15-16}$ different size QDs ${ }^{17-18,22}$ also lead to changes in band alignment effects. Hyun et al. ${ }^{22}$ reported that changing the QD size also changes the energy level of the conduction band. However, the performance enhancement mechanism of band alignment in solar cells is still open to debate. Ning et al. ${ }^{8}$ suggests that it is a result of an extended depletion region, while Chuang et al. ${ }^{2}$ ascribes it to the formation of an interface dipole.

This paper focuses on controlling the coupling between QDs using a combination of QD band gap and surface chemistry to create an optimized band. Kramer et al. ${ }^{18}$ reported a similar quantum funnel CQD solar cell using large band gap PbS as a blocking layer at the rear contact with PCE of $2.7 \%$. In this design there was good conduction band alignment but poor valence band alignment as the large band gap $\mathrm{PbS}$ gave rise to a deeper valence band position. ${ }^{22}$. In this work a thin PbS layer with a smaller band gap is inserted between the PbS absorber and the gold cathode to act as a hole transport layer using a combination of different band gaps and ligand treatment to optimize band alignment as shown in Figure 1. This paper optimizes both absorber and HTL layers devices to improve PCE. 

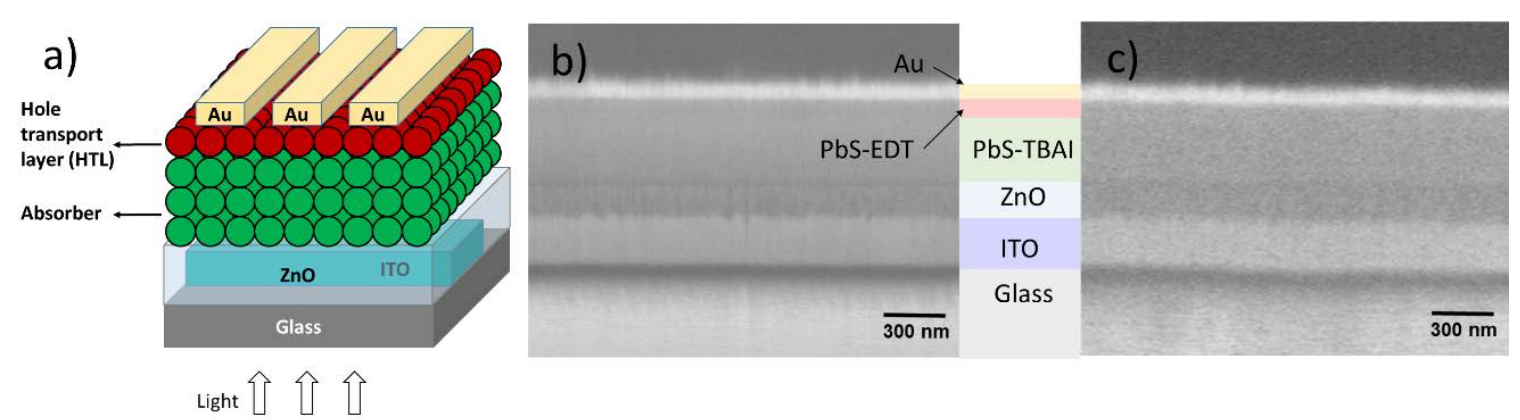

Figure 1a) The architecture of colloidal quantum dot solar cells used in this work. b) Cross-sectional SEM images of the optimized device. c) Cross-sectional SEM images of the reference device. The thickness for each layer is $210 \mathrm{~nm}$ for ITO, $150 \mathrm{~nm}$ for ZnO, 290 nm for PbS-TBAI, 70 nm for PbS-EDT, and 50 nm for Au.

\section{EXPERIMENT}

Synthesis of colloidal PbS QDs. Oleic-acid-capped PbS QDs were synthesized using Schlenk line techniques following the methods reported elsewhere. ${ }^{13,23} 1.30 \mathrm{eV} \mathrm{PbS}$ was synthesized as follows. Lead oxide (0.46 g) was dissolved in the mixture of 1-octadecene (10 g) and oleic acid (OA, $1.5 \mathrm{~g}$ ) by stirring. The solution was degassed at $105{ }^{\circ} \mathrm{C}$ under vacuum until the solution was totally clear and without bubbles. Separately, hexamethyldisilathiane $(210 \mu \mathrm{L})$ and 1-octadecene $(5 \mathrm{~g})$ were mixed as the sulphur precursor, which was injected rapidly into the lead precursor solution under Argon. After $30 \mathrm{~s}$, the reaction was cooled down in a water bath to room temperature. PbS QDs were precipitated by adding acetone, followed by centrifugation. Next, a methanol and hexane mixture was used to wash the QDs twice, and the QD precipitant was finally dissolved in octane (50 mg mL ${ }^{-1}$ ). Different band gap PbS QDs were synthesized in the same way by varying the injection temperatures and amount of oleic acid used. ${ }^{24}$ 
Synthesis of ZnO nanoparticles. The synthesis of $\mathrm{ZnO}$ nanoparticles was adapted from the literature. ${ }^{25-26}$ Zinc acetate dehydrate (2.95 g) was dissolved in methanol (125 $\mathrm{mL}$ ) by stirring at $60{ }^{\circ} \mathrm{C}$. Potassium hydroxide ( $1.50 \mathrm{~g}$ ) dissolved in $65 \mathrm{~mL}$ methanol was added, dropwise, into the zinc acetate dihydrate solution. The solution was stirred at 60 ${ }^{\circ} \mathrm{C}$ for $2 \mathrm{~h}$, then washed using dispersion and centrifuge induced precipitation with methanol three times. Finally, the $\mathrm{ZnO}$ nanoparticles were dissolved in a chloroformmethanol mixture at a concentration of about $100 \mathrm{mg} \mathrm{mL}^{-1}$.

Device fabrication. Indium tin oxide (ITO) coated glass substrates were cleaned by sonication in Decon-90, deionized water, acetone, and isopropanol, followed by an oxygen plasma treatment for $3 \mathrm{~min}$. The substrates were coated with $\mathrm{ZnO}$ on a spin coater at $2000 \mathrm{rpm}$. Subsequently PbS QD layers were spin-coated at 2000 rpm layer-by-layer using the solid state ligand exchange developed by Chuang et $\mathrm{al}^{2}$. $15 \mathrm{mg} / \mathrm{mL}$ TBAImethanol solution was coated on the PbS film for 1 min before spinning, which was subsequently washed away by 5 drop methanol. EDT-acetonitrile solution was dropped while the film was spinning, followed by 5 drop acetonitrile to rinse it. The thickness of each TBAI and EDT layer is $29 \mathrm{~nm}$ and $35 \mathrm{~nm}$ respectively as determined by using crosssectional SEMimages as can be seen in Figure 1 b\&c. The combination of band gaps from absorber table $(1.52 \mathrm{eV}, 1.37 \mathrm{eV}$, and $1.30 \mathrm{eV})$ and $\mathrm{HTL}$ table $(1.38 \mathrm{eV}, 1.20 \mathrm{eV}$, $1.12 \mathrm{eV}$, and $1.00 \mathrm{eV}$ ) were conducted. The optimized device was fabricated with 10 TBAI layers with $1.38 \mathrm{eV} \mathrm{PbS}$ in the front, and 2 EDT layers with $1.12 \mathrm{eV} \mathrm{PbS}$ at the back. The reference device was built up with only $1.38 \mathrm{eV} \mathrm{PbS}$ in both TBAI and EDT layers for the same thickness $(290 \mathrm{~nm}+70 \mathrm{~nm})$. Au electrode evaporation was conducted on the films in a glove box at $0.1 \mathrm{~nm} / \mathrm{s}$ until reaching a thickness of $50 \mathrm{~nm}$. 
Optoelectronic characterization. All solar cell devices were loaded into a sealed testing chamber with $\mathrm{N}_{2}$ continually purged through testing. I-V curves were measured using a Keithley 2400 Source Meter. An AM 1.5 solar spectrum illumination was produced by a Newport solar simulator (model 67005) with an AM 1.5 filter. The power density of the light source was calibrated with a calibrated Si reference cell from ABET Technologies (model 15151). Backward voltage scan data between 1 and -0.4 V was used in this paper. To avoid overestimation of performance due to edge effects, a slightly smaller mask $\left(1.28 \mathrm{~mm}^{2}\right)$ than the actual area of the cathode and anode $\left(2.00 \mathrm{~mm}^{2}\right)$ was used when testing the I-V curves. EQE was tested with an Oriel 1/8 m monochromator coupled to a quartz tungsten halogen lamp. $410 \mathrm{~nm}, 600 \mathrm{~nm}$, and $1000 \mathrm{~nm}$ filters were used to remove second order grating effects. Calibrated photodiodes from Newport (818UV and 918-IR) were used to measure photon flux at the device. The beam was chopped at a frequency of $220 \mathrm{~Hz}$ by a Bentham 218 chopper controller and a Xenon lamp simultaneously shone on the devices. Solar cell devices were placed under constant $\mathrm{N}_{2}$ flow and connected to a Bentham 227 pre-amplifier, a Bentham 225 lock-in amplifier, and a Keithley6485 picoammeter. Open circuit voltage decay was used to determine the carrier lifetime by applying a LED pulse on the devices and recording the $\mathrm{V}_{\text {oc }}$ decay on an oscilloscope. ${ }^{27}$

Band position characterization. The band gap of CQDs colloids and thin films was determined using a Cary Varian 4000 UV-vis-NIR spectrophotometer. Fermi level position was measured using a KP Technology Scanning Kelvin Probe System 2001. For each sample, 25 measurements were taken at 3 arbitrarily chosen points on the sample. The instrument was calibrated using aluminum and gold thin films. The conduction band 
edge positions of different PbS QD films was determined by cyclic voltammetry in a double Faraday cage with an Autolabpotentiostat (Metrohm-Autolab BV, Netherlands) using a three electrode system at $298 \mathrm{~K}$. PbS QD films spin-coated on ITO were used as the working electrodes, while $\mathrm{Ag} / \mathrm{AgCl}$ in saturated $\mathrm{KCl}$ was used as the reference electrode and a platinum foil the counter electrode. All the measurements are carried out in acetonitrile (99.9\%, Sigma Aldrich) with $0.1 \mathrm{M} \mathrm{Bu}_{4} \mathrm{NClO}_{4}$ (Fluka) as the supporting electrolyte. Before the measurements, the solution was degassed with a $\mathrm{N}_{2}$ flow for 5 min to remove $\mathrm{O}_{2}$, and atmosphere of $\mathrm{N}_{2}$ was maintained during the experiment. Valence band edge positions was calculated based on the optical band gaps from absorbance. Scanning range is between $-2.0 \mathrm{~V}$ to $1.4 \mathrm{~V}$ at a scan rate of $100 \mathrm{mV} \mathrm{s}^{-1}$. The applied potential starts from $0.0 \mathrm{~V}$ and follows a negative-going direction at first.

\section{RESULTS AND DISCUSSION}

A series of devices were first fabricated to optimize the absorbers' band gap using 1.52 $\mathrm{eV}, 1.37 \mathrm{eV}$, and $1.30 \mathrm{eV}$ CQDs modified by TBAI as absorbers respectively, with 1.20 eV CQDs modified by EDT as hole transport layers as shown in Table 1 . The 1.37eV/1.20eV (absorbers/HTL) CQD device demonstrated the best device performance. Table 1 Absorber band gap optimization: devices presented with mean and standard deviation.

\begin{tabular}{|l|l|l|l|l|}
\hline $\begin{array}{l}\text { Absorber/HTL } \\
\text { Band gap }\end{array}$ & $\mathrm{J}_{\mathrm{sc}}\left(\mathrm{mA} \mathrm{cm}{ }^{-2}\right)$ & $\mathrm{V}_{\text {oc }}(\mathrm{V})$ & FF & Efficiency \\
\hline $1.52 \mathrm{eV} / 1.20 \mathrm{eV}$ & $21.73 \pm 1.51$ & $0.58 \pm 0.004$ & $0.51 \pm 0.02$ & $6.47 \pm 0.54 \%$ \\
\hline $1.37 \mathrm{eV} / 1.20 \mathrm{eV}$ & $24.65 \pm 3.09$ & $0.60 \pm 0.008$ & $0.60 \pm 0.05$ & $8.97 \pm 0.56 \%$ \\
\hline $1.30 \mathrm{eV} / 1.20 \mathrm{eV}$ & $26.97 \pm 0.38$ & $0.49 \pm 0.004$ & $0.56 \pm 0.02$ & $7.58 \pm 0.41 \%$ \\
\hline
\end{tabular}


The optimum absorber band gap was found to be $1.37 \mathrm{eV}$. The EDT-modified HTL's band gap was next optimized. Devices with absorber/HTL combinations of $1.38 \mathrm{eV} / 1.38 \mathrm{eV}, 1.37 \mathrm{eV} / 1.20 \mathrm{eV}, 1.38 \mathrm{eV} / 1.12 \mathrm{eV}$, and $1.38 \mathrm{eV} / 1.00 \mathrm{eV}$ were fabricated and compared, as Table 2 shows. With the HTL band gap decreasing, the PCE peaked at 1.12 $\mathrm{eV}$ with champion efficiency of $9.33 \pm 0.50 \%$.

Table 2HTL band gap optimization: devices presented with mean and standard deviation.

\begin{tabular}{|l|l|l|l|l|}
\hline $\begin{array}{l}\text { Absorber/HTL } \\
\text { Band gap }\end{array}$ & $\mathrm{J}_{\mathrm{sc}}\left(\mathrm{mA} \mathrm{cm}{ }^{-2}\right)$ & $\mathrm{V}_{\text {oc }}(\mathrm{V})$ & FF & Efficiency \\
\hline $1.38 \mathrm{eV} / 1.38 \mathrm{eV}$ & $31.68 \pm 4.51$ & $0.59 \pm 0.008$ & $0.48 \pm 0.06$ & $8.86 \pm 0.26 \%$ \\
\hline $1.37 \mathrm{eV} / 1.20 \mathrm{eV}$ & $24.65 \pm 3.09$ & $0.60 \pm 0.008$ & $0.60 \pm 0.05$ & $8.97 \pm 0.56 \%$ \\
\hline $1.38 \mathrm{eV} / 1.12 \mathrm{eV}$ & $25.30 \pm 2.11$ & $0.59 \pm 0.007$ & $0.63 \pm 0.02$ & $9.33 \pm 0.50 \%$ \\
\hline $1.38 \mathrm{eV} / 1.00 \mathrm{eV}$ & $21.49 \pm 0.47$ & $0.53 \pm 0.010$ & $0.53 \pm 0.01$ & $6.03 \pm 0.40 \%$ \\
\hline
\end{tabular}

To understand why the optimized $1.38 \mathrm{eV} / 1.12 \mathrm{eV}$ device achieved the highest PCE its optoelectronic properties are now compared to a reference device's with an absorber/HTL combination of $1.38 \mathrm{eV} / 1.38 \mathrm{eV}$. The effect of different QD sizes and ligands on band gap was determined using UV-visible absorption spectroscopy and is presented in supplementary information (Figure S 1).

Figure 2a compares the current-voltage $(\mathrm{I}-\mathrm{V})$ curves of optimized and reference devices. The mean PCE is $9.33 \%$ with standard deviation of $0.50 \%$ for the optimized devices while the reference's mean PCE is $8.86 \%$ with standard deviation of $0.26 \%$. With prolonged exposure in an air atmosphere both optimized and reference performance become stable after the first 10 days. As previously reported, ${ }^{2}$ the TBAI/EDT device 
structure is air stable. After three months of storage in the air and in the dark (Figure 2c), the PCE did not show any obvious decrease. Besides PCE, the open circuit voltage (Figure S 2b) and fill factor (Figure 2b) are also higher in the optimized device than that of the reference. However, the short circuit current $\left(\mathrm{J}_{\mathrm{sc}}\right.$, Figure $\left.\mathrm{S} 2 \mathrm{2a}\right)$ in the optimized device is lower.
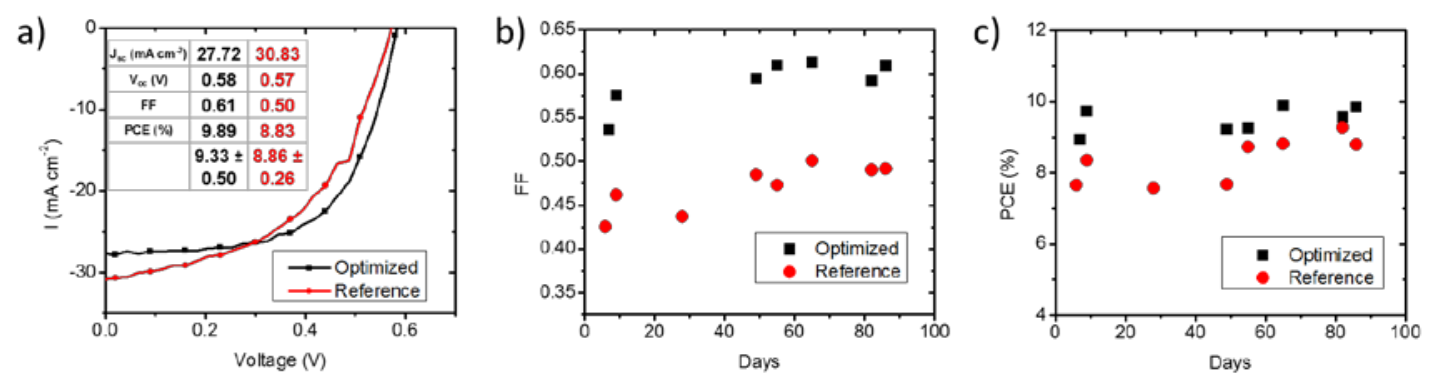

Figure 2 Optimized and reference devices’ photovoltaic performances: a) I-V curves, b) Variation of fill factor and c) PCE over 80 days

We hypothesize that the higher $\mathrm{V}_{\text {oc }}$ and FF observed is related to the different band structures which result from different QDs' size and ligand modification for the absorber and HTL which results from using TBAI and EDT respectively. To test this a combination of Kelvin probe and cyclic voltammetry is used.

Figure 3a shows the position of conduction and valence bands for the different sized $\mathrm{PbS}$ with different ligands. Comparing the $1.36 \mathrm{eV}$-TBAI PbS and $1.36 \mathrm{eV}$-EDT PbS it is clear that EDT modification raises the band gap position relative to the vacuum. As observed by Chuang et al. ${ }^{2}$, this blocks the flow of electrons and enhances hole extraction at the positive electrode. By decreasing the HTL PbS band-gap the conduction band 
becomes deeper and the valence band shallower. Thus further enhancing the hole extracting properties of the EDT layer.

Fermi level position measured by Kelvin probe is presented in Figure 3c\&d. It is clear that the $1.38 \mathrm{eV}$-EDT PbS thin film has a deeper Fermi level $(-4.84 \mathrm{eV})$ than $1.38 \mathrm{eV}$ TBAI (-4.79 eV) layer. This indicates more p-type behavior relative to the valence band positon. If a smaller band gap $1.12 \mathrm{eV}$ EDT layer is used, the Fermi level is further lowered to $-4.87 \mathrm{eV}$ indicating even more p-type layer, considering its valence band position changes to be shallower as shown in Figure 3a.

When the absorber and HTL layers are combined together as shown in Figure 3d, the combined layers' fermi level lies between the original isolated TBAI layers' and EDT layers' fermi levels because of the fermi level equilibrium of these two layers, as can be seen in Figure 3d and Table S 3. Thus the optimized layers have a deeper fermi level than the reference layers, and also a more p-type character. As open circuit voltage is significantly influenced by the quasi-Fermi level differences between the $\mathrm{ZnO}$ and $\mathrm{PbS}$ layers, the deeper Fermi level in the PbS layers leads to larger $\mathrm{V}_{\text {oc }}$ as can be seen in Figure S 2b. ${ }^{13,28}$

Combining the fermi level and band edge structures analysis, the full device band structures can be seen in Figure 3b. When comparing the 1.38eV-EDT and 1.12eV-EDT HTL layers, the latter showed better valence band offset and deeper fermi level without losing the advantages of blocking electrons, resulting in better HTLs here. 


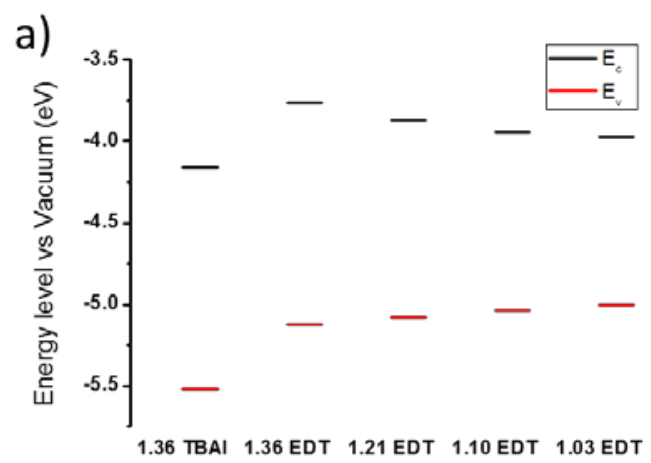

b)

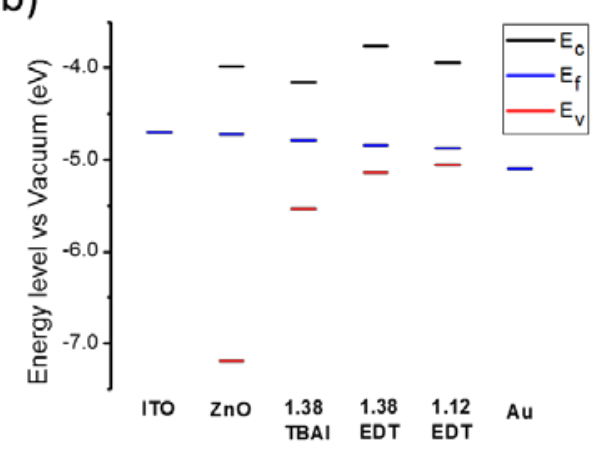

c)

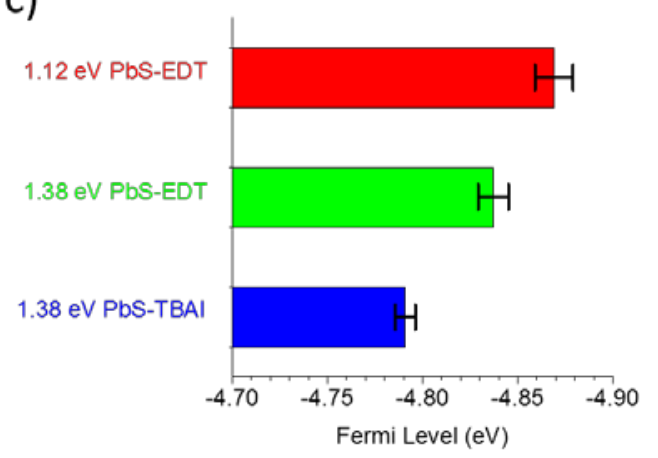

d)

$1.38 \mathrm{eV}$ PbS TBAII

$1.12 \mathrm{eV}$ PbS EDT
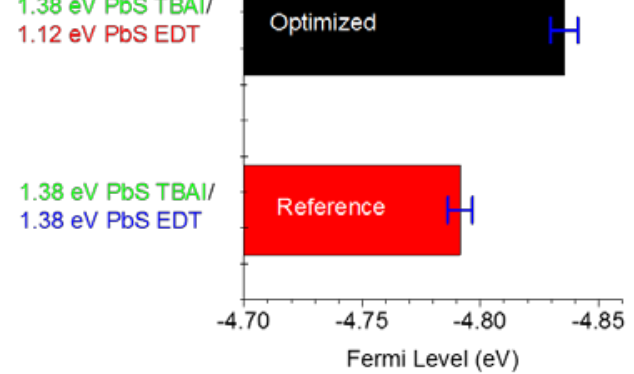

Figure 3 Band structures from cyclic voltammetry and fermi level measurements using Kelvin probe on different films: a) band structures of different PbS films, b) isolated materials' band structures in the reference and optimized devices, c) fermi levels of six layer PbS films with only one ligand (TBAI: $174 \mathrm{~nm}$, and EDT: 210nm), and d) fermi levels of 8 layer PbS films (244 nm) consisting of 6 TBAI layer (174 nm) and 2 EDT layer $(70 \mathrm{~nm})$.

There is a trade-off between gaining valence band alignment advantages and losing conduction band alignment advantages as shown in Figure 3a. Therefore, careful selection of band gap and Fermi level combination is required to optimize devices. Compared to the $1.37 \mathrm{eV} / 1.20 \mathrm{eV}$ device, $\mathrm{V}_{\text {oc }}$ for the case of a smaller absorber band gap $(1.30 \mathrm{eV} / 1.20 \mathrm{eV})$ is lower due to a small quasi fermi level difference of $\mathrm{PbS}$ and 
ZnOlayers (Figure $4 \mathrm{~b} \& \mathrm{c}$ ). $\mathrm{J}_{\mathrm{sc}}$ and $\mathrm{FF}$ are comparable to the $1.37 \mathrm{eV} / 1.20 \mathrm{eV}$ case. Comparing $1.52 \mathrm{eV} / 1.20 \mathrm{eV}$ with $1.37 \mathrm{eV} / 1.20 \mathrm{eV}$ the $\mathrm{V}_{\text {oc }}$ is comparable, but $\mathrm{FF}$ and $\mathrm{J}_{\mathrm{sc}}$ are reduced because of the poorer conduction band alignment which block electrons between the two $\mathrm{PbS}$ layers, leading to increased recombination (and hence slightly depressing $\mathrm{V}_{\text {oc }}$ too). Therefore, an appropriate band gap combination is necessary for high PCE. In combinations of $1.37 \mathrm{eV} / 1.20 \mathrm{eV}$, EDT modification results in a shallower conduction edge position in the rear PbS-EDT layer, which creates an energy barrier to block electron transport to the Au electrode. Therefore, such an optimally aligned band structure for optimizing absorbers (Figure $4 \mathrm{~b}$ ) formed with the contribution by both different ligand modification and different quantum confinement effects on band alignment. Further optimization on HTL layers was conducted, the diagram of which is shown in Figure S 3, and the best device $1.38 \mathrm{eV} / 1.12 \mathrm{eV}$ was demonstrated.

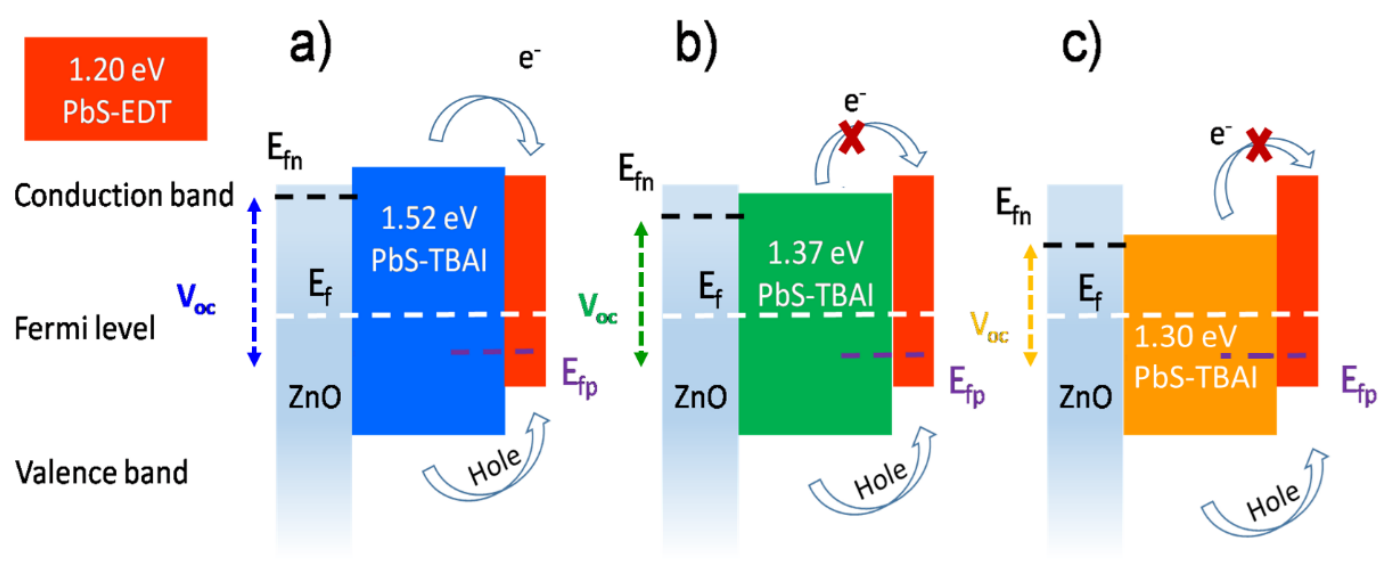

Figure 4 Band diagram of devices for absorbers' band gap optimization: a) 1.52 eV/1.20 $\mathrm{eV}$, b) $1.37 \mathrm{eV} / 1.20 \mathrm{eV}$, and c) $1.30 \mathrm{eV} / 1.20 \mathrm{eV}$.

This better band alignment in the optimized device can also be implied by its longer carrier lifetime in measurements in the decay of open circuit voltage. Under different 
light density, the optimized device's carrier lifetime is always longer than in the counterpart reference devices as shown in Figure 5a. Under air mass (AM) 1.5, the optimized device's carrier lifetime is $4.6 \mu$ s, while that of the reference device is just 2.8 $\mu \mathrm{s}$. The better band alignment structure lowers the barrier for carrier transport and hence reduces the probability of carrier recombination.

Furthermore, the incident light intensity dependence of short circuit current was studied (Figure 5b).

$$
J_{s c} \propto I^{\alpha}
$$

If $\mathrm{J}_{\mathrm{sc}}$ is observed to change linearly with incident light intensity, it can be said that the electron and hole extraction are balanced. ${ }^{28-29}$ Deviation of $\alpha$ from 1 implies the formation of space charge depletion region. ${ }^{29-30}$ For the optimized device and reference device, both $\alpha$ values are near 0.90 , indicating that there is a small, but similar, space charge limiting effect in both cases. This similarity in the quality of the devices makes the electrical property comparison more meaningful. 

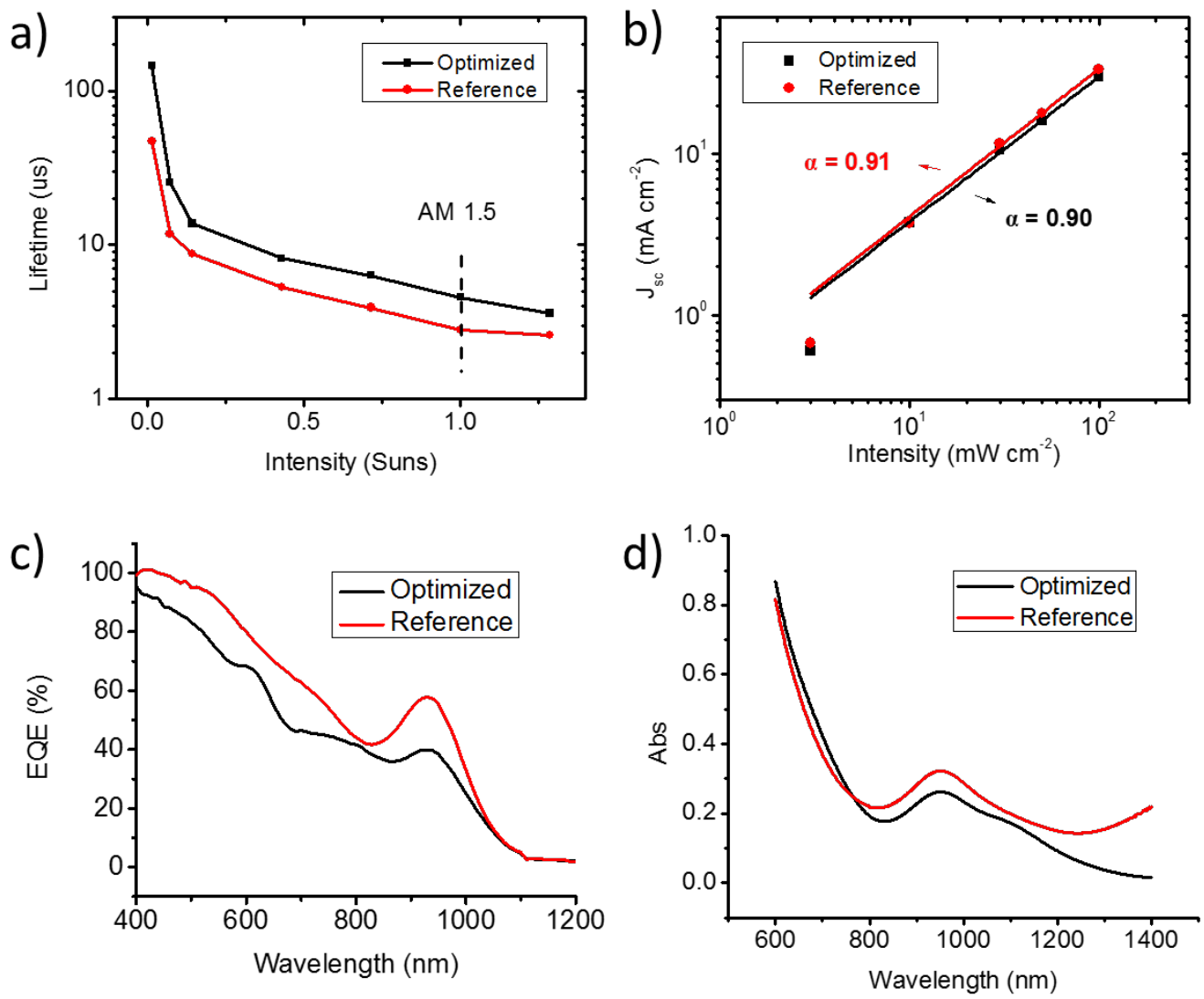

Figure 5 Optimized and reference devices’ electrical performance comparison: a) carrier lifetime, b) light density dependence of $\mathrm{J}_{\mathrm{sc}}, \mathrm{c}$ ) EQE, and d) UV-vis light absorption.

To research whether there is more light absorption contributing to the PCE enhancement, external quantum efficiency (EQE) and UV-visible absorption (UV-vis) measurements were conducted on the optimized and reference devices and films. In Figure 5c, it can be seen that the reference showed higher EQE than the optimized device, and the same peaks are found in both. The integrated $\mathrm{J}_{\mathrm{sc}}$ from EQE $\left(22.4 \mathrm{~mA} \mathrm{~cm}{ }^{-2}\right.$ for optimized devices; $26.3 \mathrm{~mA} \mathrm{~cm}{ }^{-2}$ for reference devices) is a bit lower than that derived from I-V curves $\left(25.3 \mathrm{~mA} \mathrm{~cm}{ }^{-2}\right.$ for optimized devices; $31.7 \mathrm{~mA} \mathrm{~cm}{ }^{-2}$ for 
reference devices in Table 2). UV-vis curves also showed less absorption on the optimized device than reference. Only a small peak around $1100 \mathrm{~nm}$ corresponding to the small band gap PbS was found in the optimized device's curve (Figure 5d), but this contributes so little that it cannot be seen on EQE. When increasing EDT layer thickness, the PCE even becomes lower (Figure S 4). Although the band gap distribution in the optimized device is more aligned with the light wavelength distribution across the solar cell, it seems the absorption depth for the infrared is beyond the active layer thickness. This further means that it is the optimal band alignment that is mainly responsible for the performance enhancement, rather than any additional absorption by smaller band gap layers.

\section{CONCLUSION}

The band gaps of Absorber and HTL layers of CQD solar cells have been optimized. It was found that a small band gap HTL layer with a more p-type-inducing ligand (EDT) resulted in the best devices $(1.38 \mathrm{eV} / 1.12 \mathrm{eV})$ with efficiency of $9.33 \pm 0.50 \%$. The reason for higher efficiencies is mainly improved band alignment at the interface between the absorber and HTL, which results from an upshift of valence band position due to narrow band gap CQDs and EDT ligands, and a lowering of the Fermi level due to oxidation. Improvements by further optimization of band gap combinations, layer thickness control, and ligand selection are expected further optimize the device so that it has more balanced hole and electron transport as well as better light absorption.

\section{ASSOCIATED CONTENT}


Supporting Information. UV-vis results, description for detailed testing conditions, cyclic voltammetry tests, and supplementary characterization results. This material is available free of charge via the Internet at http://pubs.acs.org.

\section{AUTHOR INFORMATION}

\section{Corresponding Author}

*Email: Andrew.watt@materials.ox.ac.uk

\section{Notes}

The authors declare no competing financial interest.

\section{ACKNOWLEDGMENTS}

We acknowledge the funding from EPSRC (Platform Grant No. EP/F048009/1 and EP/J009857/1). N. Z. acknowledges the funding from China Scholarship Council (CSC No. 201508060203).

\section{REFERENCES}

1. $\quad$ Labelle, A. J.; Thon, S. M.; Masala, S.; Adachi, M. M.; Dong, H.; Farahani, M.; Ip, A. H.; Fratalocchi, A.; Sargent, E. H. Colloidal Quantum Dot Solar Cells Exploiting Hierarchical Structuring. Nano Lett. 2015,15 (2), 1101-1108.

2. Chuang, C. H. M.; Brown, P. R.; Bulovic, V.; Bawendi, M. G. Improved Performance and Stability in Quantum Dot Solar Cells through Band Alignment Engineering. Nat. Mater. 2014,13 (8), 796-801. 
3. Wang, Y.; Suna, A.; Mahler, W.; Kasowski, R. Pbs in Polymers - from Molecules to Bulk Solids. J. Chem. Phys. 1987,87 (12), 7315-7322.

4. $\quad$ Moreels, I.; Lambert, K.; Smeets, D.; De Muynck, D.; Nollet, T.; Martins, J. C.; Vanhaecke, F.; Vantomme, A.; Delerue, C.; Allan, G.; Hens, Z. Size-Dependent Optical Properties of Colloidal PbS Quantum Dots. ACS Nano 2009,3 (10), 3023-3030.

5. $\quad$ McDonald, S. A.; Konstantatos, G.; Zhang, S. G.; Cyr, P. W.; Klem, E. J. D.; Levina, L.; Sargent, E. H. Solution-Processed PbS Quantum Dot Infrared Photodetectors and Photovoltaics. Nat. Mater. 2005,4 (2), 138-U14.

6. Kramer, I. J.; Sargent, E. H. The Architecture of Colloidal Quantum Dot Solar Cells: Materials to Devices. Chem. Rev. 2014,114 (1), 863-882.

7. Research Cell Efficiency Records by National Renewable Energy Laboratory. http://www.nrel.gov/ncpv/images/efficiency_chart.jpg (accessed 29/05/2016).

8. $\quad$ Ning, Z. J.; Voznyy, O.; Pan, J.; Hoogland, S.; Adinolfi, V.; Xu, J. X.; Li, M.; Kirmani, A. R.; Sun, J. P.; Minor, J.; Kemp, K. W.; Dong, H. P.; Rollny, L.; Labelle, A.; Carey, G.; Sutherland, B.; Hill, I. G.; Amassian, A.; Liu, H.; Tang, J.; Bakr, O. M.; Sargent, E. H. Air-Stable N-Type Colloidal Quantum Dot Solids. Nat. Mater. 2014,13 (8), 822-828.

9. $\quad$ Ning, Z. J.; Dong, H. P.; Zhang, Q.; Voznyy, O.; Sargent, E. H. Solar Cells Based on Inks of N-Type Colloidal Quantum Dots. ACS Nano 2014,8 (10), 10321-10327. 
10. Kim, S.; Noh, J.; Choi, H.; Ha, H.; Song, J. H.; Shim, H. C.; Jang, J.; Beard, M. C.; Jeong, S. One-Step Deposition of Photovoltaic Layers Using Iodide Terminated PbS Quantum Dots. J. Phys. Chem. Lett. 2014,5 (22), 4002-4007.

11. Zhitomirsky, D.; Voznyy, O.; Levina, L.; Hoogland, S.; Kemp, K. W.; Ip, A. H.; Thon, S. M.; Sargent, E. H. Engineering Colloidal Quantum Dot Solids within and Beyond the Mobility-Invariant Regime. Nat. Commun. 2014,5, 1-7.

12. Tang, J.; Kemp, K. W.; Hoogland, S.; Jeong, K. S.; Liu, H.; Levina, L.; Furukawa, M.; Wang, X. H.; Debnath, R.; Cha, D. K.; Chou, K. W.; Fischer, A.; Amassian, A.; Asbury, J. B.; Sargent, E. H. Colloidal-Quantum-Dot Photovoltaics Using Atomic-Ligand Passivation. Nat. Mater. 2011,10 (10), 765-771.

13. Neo, D. C. J.; Cheng, C.; Stranks, S. D.; Fairclough, S. M.; Kim, J. S.; Kirkland, A. I.; Smith, J. M.; Snaith, H. J.; Assender, H. E.; Watt, A. A. R. Influence of Shell Thickness and Surface Passivation on PbS/CdS Core/Shell Colloidal Quantum Dot Solar Cells. Chem. Mater. 2014,26 (13), 4004-4013.

14. Ip, A. H.; Thon, S. M.; Hoogland, S.; Voznyy, O.; Zhitomirsky, D.; Debnath, R.; Levina, L.; Rollny, L. R.; Carey, G. H.; Fischer, A.; Kemp, K. W.; Kramer, I. J.; Ning, Z. J.; Labelle, A. J.; Chou, K. W.; Amassian, A.; Sargent, E. H. Hybrid Passivated Colloidal Quantum Dot Solids. Nat. Nanotechnol. 2012,7 (9), 577-582.

15. Brown, P. R.; Kim, D.; Lunt, R. R.; Zhao, N.; Bawendi, M. G.; Grossman, J. C.; Bulovic, V. Energy Level Modification in Lead Sulfide Quantum Dot Thin Films through Ligand Exchange. ACS Nano 2014,8 (6), 5863-5872. 
16. Santra, P. K.; Palmstrom, A. F.; Tanskanen, J. T.; Yang, N.; Bent, S. F. Improving Performance in Colloidal Quantum Dot Solar Cells by Tuning Band Alignment through Surface Dipole Moments. J. Phys. Chem. C 2015,119 (6), 2996-3005.

17. Gao, J. B.; Luther, J. M.; Semonin, O. E.; Ellingson, R. J.; Nozik, A. J.; Beard, M. C. Quantum Dot Size Dependent J-V Characteristics in Heterojunction $\mathrm{ZnO} / \mathrm{PbS}$ Quantum Dot Solar Cells. Nano Lett. 2011,11 (3), 1002-1008.

18. Kramer, I. J.; Levina, L.; Debnath, R.; Zhitomirsky, D.; Sargent, E. H. Solar Cells Using Quantum Funnels. Nano Lett. 2011,11 (9), 3701-3706.

19. Yang, Z.; Janmohamed, A.; Lan, X.; Garcia de Arquer, F. P.; Voznyy, O.; Yassitepe, E.; Kim, G.-H.; Ning, Z.; Gong, X.; Comin, R. Colloidal Quantum Dot Photovoltaics Enhanced by Perovskite Shelling. Nano Lett. 2015,15 (11), 7539-7543.

20. Hanrath, T. Colloidal Nanocrystal Quantum Dot Assemblies as Artificial Solids. J. Vac. Sci. Technol. A 2012,30 (3), 030802.

21. Klimov, V. I., Nanocrystal Quantum Dots. CRC Press: Boca Raton, 2009.

22. Hyun, B. R.; Zhong, Y. W.; Bartnik, A. C.; Sun, L. F.; Abruna, H. D.; Wise, F. W.; Goodreau, J. D.; Matthews, J. R.; Leslie, T. M.; Borrelli, N. F. Electron Injection from Colloidal PbS Quantum Dots into Titanium Dioxide Nanoparticles. ACS Nano 2008,2 (11), 2206-2212.

23. Hines, M. A.; Scholes, G. D. Colloidal PbS Nanocrystals with Size-Tunable nearInfrared Emission: Observation of Post-Synthesis Self-Narrowing of the Particle Size Distribution. Adv. Mater. 2003,15 (21), 1844-1849. 
24. Zhang, J.; Crisp, R.; Gao, J.; Kroupa, D. M.; Beard, M. C.; Luther, J. M. Synthetic Conditions for High-Accuracy Size Control of Pbs Quantum Dots. J. Phys. Chem. Lett. 2015,6 (10), 1830-1833.

25. Pacholski, C.; Kornowski, A.; Weller, H. Self-Assembly of ZnO: From Nanodots, to Nanorods. Angew. Chem. Int. Ed. Engl. 2002,41 (7), 1188-1191.

26. Willis, S. M.; Cheng, C.; Assender, H. E.; Watt, A. A. R. The Transitional Heterojunction Behavior of PbS/ZnO Colloidal Quantum Dot Solar Cells. Nano Lett. 2012,12 (3), 1522-1526.

27. Lederhandler, S.; Giacoletto, L. Measurement of Minority Carrier Lifetime and Surface Effects in Junction Devices. Proc. I.R.E. 1955,43 (4), 477-483.

28. Zhao, N.; Osedach, T. P.; Chang, L. Y.; Geyer, S. M.; Wanger, D.; Binda, M. T.; Arango, A. C.; Bawendi, M. G.; Bulovic, V. Colloidal PbS Quantum Dot Solar Cells with High Fill Factor. ACS Nano 2010,4 (7), 3743-3752.

29. Koster, L. J. A.; Mihailetchi, V. D.; Xie, H.; Blom, P. W. M. Origin of the Light Intensity Dependence of the Short-Circuit Current of Polymer/Fullerene Solar Cells. Appl. Phys. Lett. 2005,87 (20).

30. $\quad$ Cheng, C.; Lee, M. M.; Noel, N. K.; Hughes, G. M.; Ball, J. M.; Assender, H. E.; Snaith, H. J.; Watt, A. A. R. Polystyrene Templated Porous Titania Wells for Quantum Dot Heterojunction Solar Cells.ACS Appl. Mater. Interfaces2014,6 (16), 14247-14252. 


\section{Table of contents}

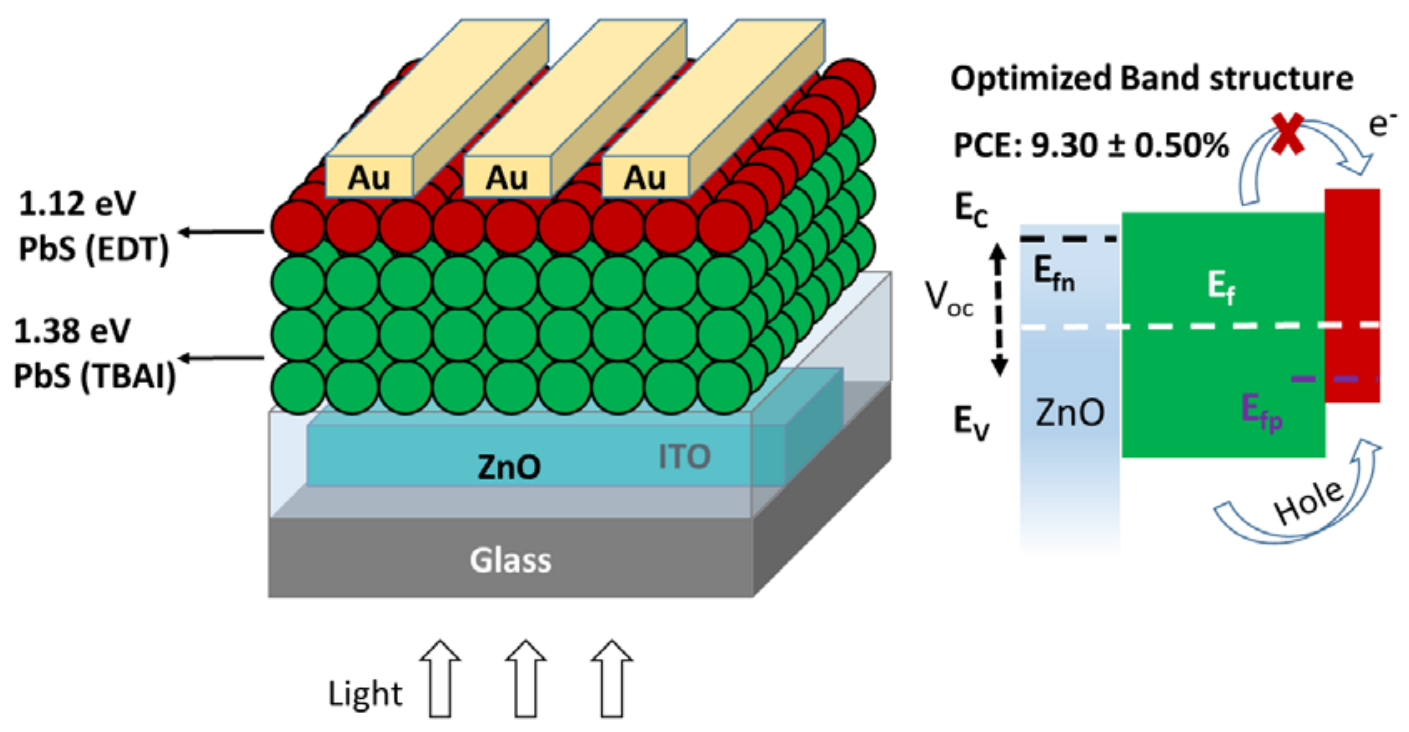

